\title{
Glucose detection in food real samples using an amperometric biosensor
}

\author{
Artur Jędrzak $^{1}$, Maria Kuznowicz ${ }^{2}$, Teofil Jesionowski ${ }^{3}$ \\ 1. Institute of Chemical Technology and Engineering, Faculty of Chemical Technology, Poznan University of \\ Technology, POLAND, Poznań, Berdychowo 4, PL-60965, E-mail: artur.jedrzak@put.poznan.pl \\ 2. Institute of Chemical Technology and Engineering, Faculty of Chemical Technology, Poznan University of \\ Technology, POLAND, Poznań, Berdychowo 4, PL-60965, E-mail: maria.m.kuznowicz@doctorate.put.poznan.pl \\ 3. Institute of Chemical Technology and Engineering, Faculty of Chemical Technology, Poznan University of \\ Technology, POLAND, Poznań, Berdychowo 4, PL-60965, E-mail: teofil.jesionowski@ put.poznan.pl
}

The goal of the research was to construct an amperometric biosensor. The biosensor was constructed on a bioinspired nanomaterial and glucose oxidase as a bioreceptor. The biosensor was used to measure glucose in real solutions of various food products.

amperometric sensor, biosensor, glucose detection, food samples, glucose oxidase, hybrid materials, nanomaterials

\section{Introduction}

Blood plasma glucose levels rise as a result of dietary carbohydrate consumption. Larger and longer rises in blood sugar levels after meals can play a role in the development of vascular complications of diabetes. The high sugar content in today's diet has been linked to a variety of chronic health problems, including obesity, diabetes, cardiovascular disease, and fatty liver disease [1].

While several methods for glucose analysis are available, such as spectrophotometry, spectrofluorometry, conductometry, gas chromatography, mass spectrometry, colorimetry, reflectance photometry, and so on, the majority of these methods require complex procedures, low reproducibility, and instability. As a result, a simple, responsive, stable, accurate, micro-volume, reproducible, and low-cost glucose analysis method must be developed as a viable alternative to existing methods [2].

Electrochemical methods are currently receiving a lot of attention because they are quick, responsive, fast, reliable, and cost-effective as compared to other methods. Enzyme-based electrodes, which combine enzyme specificity with the high sensitivity of electrochemical instruments, are now widely used in clinical diagnostics and food analysis. Biosensor systems with higher materials sensitivity, stability, selectivity, and durability are appealing alternatives among biosensors for determining the level of glucose in various media such as commercial samples (drinks, glucose-based supplements juices, fruits) or body fluids (blood plasma, whole blood) [3].

The immobilization of the widely used glucose oxidase ( $\mathrm{GOx}$ ) onto the electrode surface is an important step in enzyme-based electrochemical glucose sensing, as it facilitates successful electron transfer at the enzyme electrode interface, which is difficult because the active core (FAD) of GOx is deeply buried within the enzyme [3].

The main goal of the research was to create a hybrid matrix. Then, glucose oxidase was immobilized on its surface. The obtained nanoplatform was characterized using physicochemical and biochemical techniques. Finally, a second-generation biosensor was constructed using an external mediator. Several electrochemical tests have been carried out to optimize its performance. Some glucose-containing compounds, as well as real solutions, were tested. The obtained results were compared with the spectrophotometric technique to more precisely check the effectiveness of the sensor. 


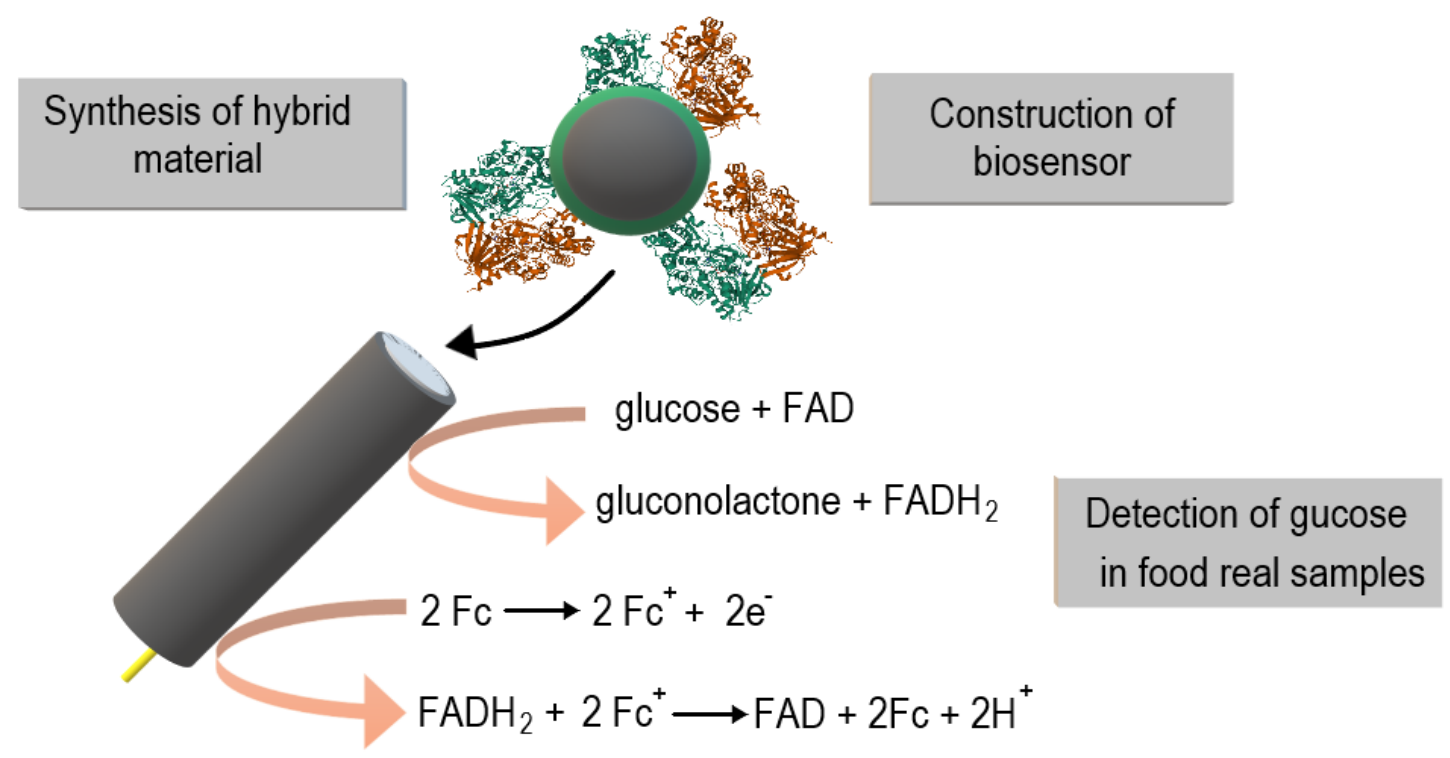

Fig.1. Schematic presentation of research work

The presented sensor was characterized by good selectivity, stability, and a wide range of linearity. The generated platform can serve as a matrix for the detection of other compounds in food when a different bioreceptor is used.

\section{Conclusion}

This paper presents an amperometric biosensor based on a hybrid material and glucose oxidase. The presented sensor was characterized by high sensitivity and selectivity. Besides, it was used with great efficiency to detect glucose in real solutions. Our proposed biosensors, which are based on hybrid micro and nanoplatforms, show promise as a biodetector in the food industry and diabetes care.

\section{Acknowledgments}

This work was financed and prepared as part of a research project supported by the National Science Center Poland, no. 2017/27/B/ST8/01506.

\section{References}

[1] Artigues M., Abellà J., Colominas S. (2017). Analytical Parameters of an Amperometric GlucoseBiosensor for Fast Analysis in Food Samples. Sensors, 17, 2620, doi:10.3390/s17112620

[2] Jędrzak A., Rębiś T., Klapiszewski Ł., Zdarta J., Milczarek G., Jesionowski T. (2018) Carbon paste electrode based on functional GOx/silica-lignin system to prepare an amperometric glucose biosensor, Sensors Actuators B: Chem., 256, 176-185. doi: 10.1016/j.snb.2017.10.079

[3] Jayanthi Kalaivani G., Suja S.K (2019) Nanomolar level sensing of glucose in food samples using glucose oxidaseconfined MWCNT-Inulin- $\mathrm{TiO}_{2}$ bio-nanocomposite, Food Chemistry, 298, 124981, doi:10.1016/j.foodchem.2019.124981

[4] Jędrzak A, Rębiś T, Kuznowicz M., Jesionowski T (2019) Bio-inspired magnetite/lignin/polydopamine-glucose oxidase biosensing nanoplatform. From synthesis, via sensing assays to comparison with others glucose testing techniques. Int. J. Biol. Macromol. 127, 677-682 doi: 10.1016/j.ijbiomac.2019.02.008 